Conclusions: The prevalence of depressive and anxious symptoms among gout patients was higher than healthy individuals. Education, disability, tophi and HRQoL were important risk factors linked to this disorder in Chinese gout population. These findings suggested medical personnel should pay more attention to the psychological health of gout patients and make objective interventions to relieve their depression and anxiety, especially those with low education level, more than two tophi, severe disability, and poor HRQoL.

Acknowledgements: This study was supported by Grants from the Chinese National Natural Science Foundation (no. 81671616 and 81471603).

Disclosure of Interest: None declared

DOI: 10.1136/annrheumdis-2017-eular.3082

\section{AB0900 SERUM URIC ACID LEVEL VARIATIONS DURING GOUT ATTACKS ARE LINKED NEITHER TO INFLAMMATION NOR TO URIC ACID FRACTIONAL EXCRETION: A PROSPECTIVE STUDY OF 35 PATIENTS}

V. Masson Behar ${ }^{1}$, C. Desthieux ${ }^{1}$, C. Aparicio ${ }^{2}$, P. Manivet ${ }^{3}$, T. Bardin ${ }^{1}$, P. Richette ${ }^{1}$, F. Lioté ${ }^{1}$, A. Ostertag ${ }^{1}$, H.-K. Ea ${ }^{1} .{ }^{1}$ Service de rhumatologie, hôpital Lariboisière, AP-HP, centre Viggo Petersen, University Paris Diderot, INSERM UMR 1132, Bioscar; ; ${ }^{2}$ Policlinique, hôpital Lariboisière, AP-HP; ${ }^{3} \mathrm{Crb}$ Bb-0033-00064, BioBanking and Analytics Systems, APHP, Paris, France

Background: Acute gout is usually associated with a decrease in serum uric acid (SUA) level but the mechanism responsible for this phenomenon remains unclear. Objectives: We aimed to investigate relationships between changes in SUA level, urinary excretion of uric acid and biochemical markers during gout attack. Methods: SUA, eGFR (estimated glomerular filtration rate), serum CRP level and urinary excretion of UA, expressed as fractional excretion of UA (FeUA), from 35 ULT (urate-lowering therapy)-free and diuretic-free gout patients were prospectively measured during acute gout attack (Tc) and intercritical (Tic) phase. In 11 patients, data were available after achievement of SUA target $(\mathrm{Tt})(<360$ $\mu \mathrm{mol} / \mathrm{l})$ under ULT. Demographics data and waist circumference (WC) were collected. Data are expressed as mean \pm SD.

Results: There were 32 men, mean age 57.9 years, mean body mass index $28.6 \mathrm{~kg} / \mathrm{m}^{2}$, and mean waist circumference $104 \mathrm{~cm}$. Overall $17.1 \%$ had type 2 diabetes, $37.1 \%$ dyslipidemia, $54.3 \%$ hypertension, $34.4 \%$ obesity, $74.3 \%$ abdominal obesity and $51.4 \%$ chronic kidney disease (CKD, 31.4\% CKD 2 and $20 \%$ CKD 3-5). Gout duration was $3.9 \pm 6.7$ years, $28.6 \%$ of patients had tophus and $31.4 \%$ gout arthropathy. SUA, eGFR and FeUA values were similar between Tc and Tic phases: SUA: $504.9+79.9$ and $507.6+89.7 \mu \mathrm{mol} / \mathrm{l} ;$ eGFR: $78.1+28.8$ and $81.4 \pm 33.8 \mathrm{ml} / \mathrm{min} / 1.73 \mathrm{~m}^{2}$; FeUA: $4.90 \pm 2.3$ and $4.45 \pm 3.3 \%$, respectively. Moreover, the FeUA values were identical between Tt, Tic and Tc in 11 patients who achieved SUA target $(296.0 \pm 37.8 \mu \mathrm{mol} / \mathrm{l})$ under ULT. CRP levels were higher at Tc $(44.8 \pm 69.4 \mathrm{mg} / \mathrm{dL})$ than Tic $(5.2 \pm 3.3 \mathrm{mg} / \mathrm{dl})$ and Tt $(4.6 \pm 1.2 \mathrm{mg} / \mathrm{dl})$. While mean SUA levels were similar between Tc and Tic, SUA levels were lower in Tc than Tic in 21 patients (mean difference: $56.9 \pm 33.7 \mu \mathrm{mol} / \mathrm{l}$ ) (figure1). In contrast, 14 patients had a SUA level higher in Tc than in Tic (mean difference: 78.6 $6 \pm 71.5$ $\mu \mathrm{mol} / \mathrm{L}$ ) (figure 1). SUA variations between Tc and Tic were not correlated with FeUA modifications in these two groups $(p=0.15)$. Similarly, SUA and FeUA variations were not correlated with CRP variations between Tc and Tic ( $p=0.29$ and $p=0.30$, respectively).

Figure 1. Variations of SUA between gout attack (TC) and intercritical (Tic) phases (Green color: patients $(\mathrm{N}=21)$ with lower SUA level at Tc than at

Tic; red color: patients ( $N=14$ ) with higher SUA level at Tc than at Tic)

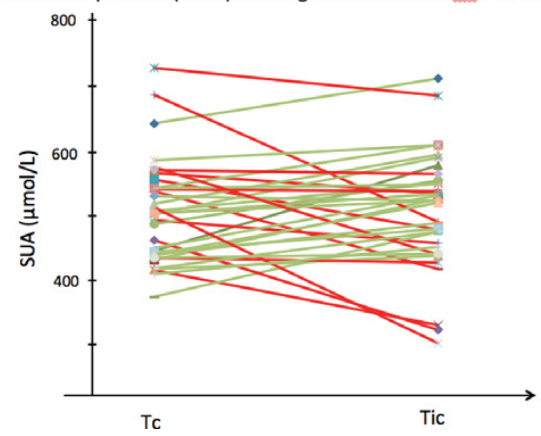

Conclusions: SUA levels during gout attack can be either higher or lower than SUA during intercritical phase. These variations are linked neither to inflammation level nor to FeUA. It would be interesting to assess the intestinal excretion of uric acid, xanthine oxidase activity and diet intake during these different gout phases.

Disclosure of Interest: None declared

DOI: 10.1136/annrheumdis-2017-eular.4808

\section{AB0901 CLINICAL SIGNIFICANCE OF URATE DEPOSITION IN TENDON: A DUAL-ENERGY CT STUDY}

Y. Eun ${ }^{1}$, I.Y. Kim ${ }^{1}$, H. Jeong ${ }^{1}$, E.-J. Park ${ }^{2}$, H. Kim ${ }^{1}$, J. Lee ${ }^{1}$, E.-M. Koh ${ }^{1}$, H.-S. Cha ${ }^{1} .{ }^{1}$ Department of Medicine, Samsung Medical Center, Seoul; ${ }^{2}$ Department of Medicine, Jeju National University Hospital, Jeju, Korea, Republic Of

Background: Dual-energy computed tomography (DECT) is advanced imaging modality that shows the deposition of monosodium urate (MSU) crystal in tissue as a color signal. The MSU crystal deposit around the symptomatic joint is considered as positive finding, but the clinical significance of urate deposition around the tendon is still unclear.

Objectives: The aim of this study was to compare the clinical characteristics and DECT findings in people with MSU crystal deposition in the joints and people with urate deposition in the tendons.

Methods: DECT was performed in 71 patients who complained of recurrent painful swelling of the joints, and 35 of them showed MSU crystal deposition in the joints on DECT. Clinical manifestation and serum uric acid level data were collected.

Results: Most of the included patients were middle-aged (mean age 50 years, SD 15) and 67 patients (94\%) were male. All patients who had MSU crystal deposition in joints on DECT had a history of typical gout attacks, and 29 patients $(81 \%)$ had a history of gout attacks among patients with urate deposition only in tendons $(p=0.011)$. The mean uric acid level of patients included in the study was as high as $7.5 \pm 2.2 \mathrm{mg} / \mathrm{dL}$. In the highest uric acid level, the absolute value was higher in patients with urate deposition in joints, but there was no statistical significance. The correlation between the gout attack site and the urate deposit sites was $91 \%$ in patients with joint involvement, but only $6 \%$ in patients without joint involvement $(p<0.001)$. There were 4 patients $(11 \%)$ who showed gouty erosion without MSU crystal deposition in joints on DECT.

Conclusions: The MSU crystal deposition in the tendon was not correlated well with clinical features, suggesting that it is more likely to be associated with artifact or asymptomatic hyperuricemia. However, in some patients, MSU crystal deposition may be observed only in the tendon, even with gouty erosion. Therefore, careful interpretation of the DECT results is necessary.

Disclosure of Interest: None declared

DOI: 10.1136/annrheumdis-2017-eular.6331

\section{Infection-related rheumatic diseases}

\section{AB0902 TUBERCULOSIS SCREENING IN PATIENTS RECEIVING BIOLOGIC THERAPY}

S. Bouden, A. Fazaa, K. Oueniche, S. Saidi, S. Kassab, S. Chekili, K. Ben Abdelghani, A. Laatar. Rheumatology, Hopital Mongi Slim, la Marsa, Tunisia

Background: The advent of biological treatments has greatly improved the management of chronic inflammatory diseases (CID). However, these biologics increase the risk of infection including the possible development of tuberculosis (TB). Screening of latent tuberculosis infection (LTBI) is therefore necessary prior to their initiation, especially in Tunisia, which is considered as a high-incidence area of TB.

Objectives: The aims of this study were to identify the prevalence of LTBI among patients candidate to initiate biologics, to analyze the tolerance of preventive therapy and to detect active TB or conversions of immunodiagnostic tests under biologics.

Methods: A retrospective study was conducted, over a period of 14 years (2002-2016). Patients with CID, candidate to initiate biological treatment, were included. The screening of LTBI was performed according to the national Tunisian guidelines. Clinical data, screening and follow-up information on biological therapy were assessed.

Results: A total of 76 patients were enrolled in the study, 32 men and 44 women with a mean age of 66 years [17-80]. Rheumatoid arthritis (RA) was the most common CID (44\%). The diagnosis of LTBI was established in 16 cases $(21 \%)$. Among them, 3 had a Tuberculin Skin Test (TST) more than $10 \mathrm{~mm}$ associated with a positive Interferon Gamma Release Assay (IGRA), 11 had only a positive TST, and 2 had only a positive IGRA. One of them had a history of pulmonary TB but adequately treated. All patients with positive screening were considered for preventive treatment. Thirteen $(81 \%)$ received an association of isoniazid-rifampicin for 3-6 months, and $3(19 \%)$ received isoniazid for 6 months. Toxicity was reported in 4 cases $(25 \%)$ : hepatotoxicity $(n=1)$, dermatologic toxicity $(n=1)$, fever $(n=1)$ and stomachache $(n=1)$. During the follow-up period, no case of reactivation has been reported among patients with LTBI. Out of the 60 patients with negative baseline screening, only 4 have been re-screened $(6 \%)$ and none had conversions in immunodiagnostic tests. However, among patients who screened negative, one case of active pulmonary TB has been reported in a woman who had an ankylosing spondylitis (AS) and who was receiving infliximab during 22 weeks. She hadn't previous TB history or new TB exposure.

Conclusions: Our study showed that the Tunisian recommendations allowed detecting a LTBI in $21 \%$ of biologic therapy candidates. The initial screening and the prophylactic treatment improve the safety of these treatments. However, we noted a low rate of re-screening, as the Tunisian guidelines do not recommend 
annual screening. Wide studies should be designed in order to evaluate the efficacy of re-screening.

References:

[1] He D, Bai F, Zhang $S$ et al. High incidence of tuberculosis infection in rheumatic diseases and impact for chemoprophylactic prevention of tuberculosis activation during biologics therapy. Clin Vaccine Immunol 2013; 20:842-847

[2] Carmona L, Gómez-Reino JJ, Rodríguez-Valverde V, et al. Effectiveness of recommendations to prevent reactivation of latent tuberculosis infection in patients treated with tumor necrosis factor antagonists. Arthritis Rheum 2005;52:1766-72

Disclosure of Interest: None declared

DOI: 10.1136/annrheumdis-2017-eular.6727

\section{AB0903 LABORATORY FINDINGS IN PATIENTS WITH CHIKUNGUNYA FEVER AND CHRONIC JOINT SYMPTOMS - A LONGITUDINAL ASSESSMENT}

A. Ranzolin ${ }^{1,2}$, R.M. Correia ${ }^{2}$, H.D. de Lima ${ }^{2}$, M.R.D.A. Freitas ${ }^{2}$, A.F.R. de Oliveira $^{2}$, A.L.B.P. Duarte ${ }^{1}$, C.D.L. Marques ${ }^{1}$, P.R.S. de Melo ${ }^{2}$, L.F. da Rocha $\mathrm{Jr}^{1,2} .{ }^{1}$ Hospital Das Clínicas of Pernambuco; ${ }^{2}$ Instituto de Medicina Integral Professor Fernando Figueira, Recife, Brazil

Background: Chikungunya Fever (CF) is an arbovirosis with a high attack rate, affecting large proportion of the population in its outbreaks $(85 \%>90 \%$ of infected are symptomatic). In general, it is recommended to carry out laboratory tests when patients reach subacute phase or show signs of severity at the beginning of the disease. There are few studies showing which laboratory results are relevant and their clinical applicability.

Objectives: To recognize the most frequent findings of laboratory tests in a cohort of patients with CF and chronic joint symptoms and to correlate laboratory results with clinical data.

Methods: Patients with diagnosis of CF (clinical and epidemiological criteria) were followed in a cohort study. Clinical data and laboratory tests were collected in a regular schedule in the first months of the disease.

Results: A total of 54 patients were enrolled during 10 months, persistent changes in some patients were recorded (table).

Table 1. Persistent laboratory findings in patients with Chikungunya Fever in subacute/chronic phases

\begin{tabular}{|c|c|}
\hline$>50 \%$ & Decreased vitamin D (53.8\%) \\
\hline $40 \%>50 \%$ & Increased CRP (43.3\%) \\
\hline $30 \%>40 \%$ & Decreased: HDL cholesterol (36.5\%), eosinophil (37.3\%), \\
\hline $20 \%>30 \%$ & $\begin{array}{l}\text { Increased: glucose }(28.3 \%) \text {, GGT }(27.4 \%), \gamma \text { globulin }(27.4 \%) \text {, glycated } \\
\text { hemoglobin }(26.4 \%) \text {, calcium (25.4\%), alkaline phosphatase }(24.5 \%), \beta \text { globulin } \\
(23.5 \%) \text {, cholesterol }(23.0 \%) \text { Decreased: total bilirubin }(20.0 \%)\end{array}$ \\
\hline $10 \%>20 \%$ & $\begin{array}{l}\text { Increased: triglycerides (17.6\%), LDH }(17.3 \%) \text {, ferritin (13.7\%), ALT (13.2\%), } \\
\text { direct bilirubin (12.0\%), a } 2 \text { globulin (11.7\%) Monocytosis (11.1\%), Limphocytosis } \\
(10.0 \%)\end{array}$ \\
\hline $5 \%>10 \%$ & $\begin{array}{l}\text { Hyperchloremia }(8.0 \%) \text { Increased: neutrophils }(7.54 \%) \text {, LDL }(5.88 \%) \text {, folic acid } \\
(5.88 \%) \text {, uric acid }(5.76 \%) \text {, platelets }(7.54 \%) \text { Decreased: CPK }(7.54 \%) \text {, albumin } \\
(5.88 \%) \text {, neutrophils }(9.43 \%) \text { Hyponatremia }(5.88 \%) \text {, }\end{array}$ \\
\hline
\end{tabular}

$\overline{C R P}=\mathrm{C}$ reactive protein, $\mathrm{GGT}=$ gamma glutamyl transferase, $\mathrm{LDH}=$ lactate dehydrogenase, $\mathrm{ALT}=$ alanine aminotransferase, $\mathrm{CPK}=$ creatine phosphokinase .

In the subacute phase, the ESR (erythrocyte sedimentation rate) correlated with number of swollen joints ( $r=0.45, p=0.03$ ), VAS (visual analogue scale) of pain $(r=0.72, p=0.0002)$, VAS patient's general health $(r=0.50 p=0.02)$, VAS by physician $(r=0.45, p=0.03)$ and with $\mathrm{HAQ}(r=0.51, p=0.01)$. In subacute phase the VAS of morning stiffness correlated with CRP $(r=0.46, p=0.02)$. In chronic phase, CRP correlated with VAS of pain $(r=0.47, p=0.02)$ and there was a reversal in the correlations between ESR and VAS of general health of the patient $(r=-0.54$, $p=0.03)$, VAS of physician $(r=-0.52, p=0.02)$, swollen joints $(r=-0.46 p=0.03)$ and $\mathrm{HAQ}(\mathrm{r}=-0.56, \mathrm{p}=0.01)$. ESR and SF-12 (mental component) were correlated $(r=0.61, p=0.01)$

Conclusions: Levels of ESR correlated with measures of pain and worsening of functional capacity in subacute phase. In chronic phase, there was reversal of this correlation, indicating that ESR does not reflect clinical worsening of patients at this stage. Further clinical studies are needed to better analize other alterations. References:

[1] Weaver SC, Lecuit M. Chikungunya virus and the global spread of a mosquito-borne disease. N Engl J Med 2015 Mar 26;372(13):1231-9.

[2] Brito CA, Sohsten AK, Leitão CC et al. Pharmacologic management of pain in patients with Chikungunya: a guideline. Rev Soc Bras Med Trop. 2016; 49(6):668-679.

Disclosure of Interest: None declared

DOI: 10.1136/annrheumdis-2017-eular.5599

\section{AB0904 FUSOBACTERIUM NECROPHORUM MASQUERADING AS NEISSERIA IN SEPTIC ARTHRITIS}

C.H. O'neill ${ }^{1}$, H. McCormick ${ }^{2}$, M. Leith ${ }^{1}$, L. McCorry ${ }^{3}$, J. McKenna ${ }^{2}$, A. Pendleton ${ }^{3}$, Y. Protaschik ${ }^{3}$, A. Loughrey ${ }^{2}$, P.V. Coyle ${ }^{2}$, D. Fairley ${ }^{2}$ ${ }^{1}$ Rheumatology; ${ }^{2}$ Microbiology; ${ }^{3}$ Belfast Health and Social Care Trust, Belfast, United Kingdom

Background: Infective arthritis with fusobacterium is rare and difficult to diagnose \& initially can be misidentified as Neisseria arthritis based on microscopy results. It is associated with Lemierre's Disease \& is important to recognise.

Objectives: To demonstrate that a "linked up" approach between culture and PCR in the analysis of joint fluids will provide timely identification of the organism and allow for appropriate antibiotic use \& to show the utility of bacterial 16S rRNA PCR in sterile site fluid analysis.

Methods: This is a case study of a pyogenic wrist infection with Fusobacterium necrophorum in the rheumatology department

Results: Our report follows a 17 year old caucasian female presenting with a history of left wrist pain, swelling and flu-like symptoms. Microscopy of joint aspirate revealed gram negative diplococci. Empirical antibiotic therapy, Ceftriaxone, was used to cover for potential gonococcus. Real-time PCR testing was negative for both gonococcus and meningococcus. However a real-time PCR assay targeting the bacterial $16 \mathrm{~S}$ ribosomal RNA gene detected bacterial DNA ${ }^{1}$. The patient did not clinically improve and further aspirate remained positive for the 16S rRNA gene target. As all joint aspirates and other specimens remained culture negative, the decision was taken to attempt bacterial 16S rRNA PCR and sequencing on DNA extracted directly from the joint aspirate. The sequences recovered were identified as $F$ necrophorum. This was eventually confirmed by anaerobic culture of the initial joint aspirate. A suspicion of Lemierre's disease (thrombophlebitis of the internal jugular vein and/or bacteraemia) was raised, however jugular venous dopplers were normal.

The outcome was favourable following guided antibiotic treatment.

Conclusions: This case illustrates that $F$. necrophorum infection may occur with unusual or disseminated presentation, but in the absence of the classical features of Lemierre's syndrome. The possibility of Neisseria was recognised early \& appropriate empirical antibiotic cover was used. This is important given the emergence of virulent meningococcal serogroup W ST11 strains causing infections with unusual presentation (including septic arthritis) in the UK². Reliance on microscopy findings alone could have led to an incorrect diagnosis of gonococcal septic arthritis - a condition with very low complication rates and excellent prognosis. In contrast, non-gonococcal septic arthritis is a medical emergency with significant morbidity and mortality ${ }^{3}$. The $F$. necrophorum infection in this case could have had fatal complications if it had been managed as a gonococcal infection. Secondly this case highlights the utility of bacterial 16S PCR and sequencing directly from a normally sterile site, allowing accurate diagnosis and appropriate treatment.

References:

[1] Yang S et al (2002)Quantitative Multiprobe PCR Assay for Simultaneous Detection and Identification to Species Level of Bacterial Pathogens J Clin Micro 40(9):3449-3454.

[2] Ladhani SN, et al(2014) Increase in Endemic Neisseria meningitidis Capsular Group W Sequence Type 11 Complex Associated With Severe Invasive Disease in England and Wales. Clinical Infectious Disease 60:578-585.

[3] Shirtliff \& Mader (2002) Acute septic arthritis. Clin Microbiol Rev. 15(4):527-44. Disclosure of Interest: None declared

DOI: 10.1136/annrheumdis-2017-eular.6272

\section{AB0905 THE EFFICACY OF TRIMETHOPRIM/SULFAMETHOXAZOLE TO PREVENT ACUTE-ONSET DIFFUSE INTERSTITIAL LUNG DISEASES WITH CONNECTIVE TISSUE DISEASE PATIENTS}

F.Y. Chen, K. Setoguchi, M. Ogawa, S. Kamei. Systemic Immunological Disease, Tokyo Metropolitan Komagome Hospital, Tokyo, Japan

Background: Acute-onset diffuse interstitial lung diseases (AoDILD) are highly mortal condition and their cause are often difficult to discriminate. Trimethoprim $80 \mathrm{mg} / \mathrm{sulfamethoxazole} 400 \mathrm{mg}$ single strength (TMP-SMX SS) has been used for Pneumocystis Pneumonia (PCP) prophylaxis and highly effective but the dose was originally developed in pediatric cancer patient's study and there is possibility that in connective tissue disease (CTD) patients, less dose can be as effective.

Objectives: The aim of this study is to examine hypothesis that taking TMP-SMX for PCP prophylaxis with CTD also have prophylactic effect against AoDILD and how about those effect in under dose patients for some reason

Methods: We retrospectively investigated data from 621 patients with TMP-SMX for PCP prophylaxis during and 43 patients who admitted for either acute respiratory failure/acute interstitial pneumonia/PCP/drug induced pneumonitis during 2004-2016 in our department.

Results: There was no single case who admitted to hospital due to AoDILD with TMP-SMX for PCP prophylaxis. There were 34 cases admitted for AoDILD and 9 cases (26\%) were dead. Among 25 cases, 5 cases developed AoDILD after TMP-SMX cessation. 70 cases ware taking under dose prophylaxis for some reasons but there was also no AoDILD case.

Conclusions: Taking TMP-SMX for PCP prophylaxis may also have prophylactic effect against AoDILD. And those effect may also exist even under dose. 